\title{
LA APLICACIÓN REAL DE LOS PRINCIPIOS OLÍMPICOS: LA CREACIÓN DEL EQUIPO OLÍMPICO DE REFUGIADOS PARA RÍO 2016 Y LA EXPERIENCIA DE UNA DEPORTISTA OLÍMPICA ESPAÑOLA ${ }^{1}$
}

\author{
Marina Vázquez Fernández \\ Universidad Autónoma de Madrid \\ marivafer@live.com \\ M. 'a Eugenia Martínez-Gorroño \\ Universidad Autónoma de Madrid \\ eugenia.martinez@uam.es
}

Fecha de recepción: Septiembre 2017

Fecha de aceptación: Enero 2018

http://dx.doi.org/10.15366/citius2018.11.1.003

Resumen:

Los principios olímpicos reflejados en la Carta Olímpica recogen la filosofía del Movimiento Olímpico con el fin de promover sociedades más respetuosas e igualitarias. El presente trabajo analiza la aplicación real de esos principios olímpicos a través de la creación del equipo olímpico de refugiados en los Juegos de Río 2016, y mediante el testimonio de la deportista olímpica española Aauri Bokesa. Para ello, se realizó un análisis documental de la Carta Olímpica, y se recabó información de ACNUR y del COI. Además, se llevó a cabo una entrevista semisestructurada de final abierto a la deportista olímpica. Los resultados de la investigación demostraron que los principios olímpicos que hacen referencia a la solidaridad, el establecimiento de una sociedad pacífica, la responsabilidad social y la no discriminación, son una realidad que se ha materializado en el equipo olímpico de refugiados. Del mismo modo, la deportista Aauri Bokesa asegura que los principios de la Carta Olímpica son, según su experiencia, una realidad en el Movimiento Olímpico actual.

Palabras clave: Juegos Olímpicos, Carta Olímpica, Principios olímpicos, Equipo Olímpico de Refugiados, Aauri Bokesa.

Title: THE REAL IMPLEMENTATION OF OLYMPIC PRINCIPLES: THE CREATION OF THE REFUGEES OLYMPIC TEAM FOR RIO 2016 AND THE EXPERIENCE OF A SPANISH OLYMPIC SPORTWOMAN

\section{Abstract:}

The Olympic principles, reflected in the Olympic Charter, gather the philosophy of the Olympic movement with the aim of promoting societies more respectful and egalitarian. This research analyzes the real application of the Olympic values through the creation of the Refugees Olympic Team in the Olympic Games in Río 2016, and according to the testimony of the Olympic Spanish sportswoman, Aauri Bokesa.

\footnotetext{
${ }^{1}$ Este artículo presenta el trabajo de investigación con cuyo proyecto se postuló para la participación en el 24th Seminario International de Estudios Olímpicos para Estudiantes Postgraduados organizado por la Academia Olímpica Internacional en septiembre del 2017. Este trabajo presentado por Marina Vázquez Fernández, dirigido por la Dra. M. ${ }^{a}$ Eugenia Martínez Gorroño permitió a Marina Vázquez disfrutar de una beca para ser uno de los dos representantes de España en el citado Seminario. Una versión de esta investigación ha sido publicada en inglés en las actas correspondientes al 24th International Seminar on Olympic Studies for Postgraduate Students. Con la publicación de esta versión en español, Citius, Altius, Fortius pretende facilitar el conocimiento de su contenido y el trabajo que desde las estructuras del Movimiento Olímpico se realizan a toda la comunidad hispanohablante.
} 
To this end, we have done a "documentary analyses" of the Olympic Charter and obtained information from UNHCR and IOC. Furthermore, an open-ended semi-structured interview to the Olympic sportswoman was carried out. The results show that the Olympic principles with reference to solidarity, a peaceful society, social responsibility and not discrimination are a reality reflected in the Refugees Olympic Team. In addition, the sportswoman Aauri Bokesa believes that the principles of the Olympic Charter are a reality in the current Olympic Movement.

Key Words: Olympic Games, Olympic Charter, Olympic principles, Refugees Olympic Team, Aauri Bokesa.

\section{Introducción}

Los principios y valores que implica el ideario olímpico pretenden sembrar e impulsar las bases para el fomento de sociedades más solidarias, pacíficas e igualitarias. Por ello, cualquier discriminación por raza, religión, política o sexo se opone a los principios del Movimiento Olímpico. La solidaridad, la alegría del esfuerzo, la responsabilidad social, el valor educativo del buen ejemplo, la no discriminación y la igualdad, forman parte de los principios fundamentales del Olimpismo que recoge la Carta Olímpica. Un ejemplo de la aplicación real de estos principios es la creación del equipo de refugiados que, bajo la bandera olímpica, participó en los Juegos de Río 2016. Este equipo es símbolo de esperanza y un tributo al valor y perseverancia de los refugiados. Por otro lado, estos principios, si bien están planteados en la Carta Olímpica, deberían ser vividos y valorados por cualquier deportista olímpico.

\section{Objeto de estudio}

La presente investigación se centra en conocer si el ideario olímpico está presente en la realidad práctica del olimpismo actual. Por ello, el presente trabajo rescata y testimonia la creación del equipo de refugiados que participó en los Juegos de Río 2016 como un reflejo del compromiso del Olimpismo con sus principios fundamentales, a fin de dar a conocer unos hechos de nuestra historia reciente que hablan por sí mismos de la vigencia de los ideales olímpicos en el momento actual. Por otra parte, y a fin de investigar igualmente sobre la verdadera praxis del ideario olímpico, hemos recabado información para conocer si los principios olímpicos son vivenciados por los deportistas. Para ello, hemos seleccionado a la atleta olímpica española, Aauri Bokesa, hija de un exiliado político y participante en varias ediciones de los Juegos Olímpicos.

\section{Objetivos}

El objetivo principal de esta investigación es conocer la aplicación real de los principios olímpicos y su puesta en práctica. En primer lugar, rescatando los hechos y las circunstancias de la creación del equipo de refugiados que bajo bandera olímpica ha participado en los Juegos de Río 2016 y, en segundo lugar, a partir de los hechos y las experiencias de la trayectoria deportiva de una deportista olímpica. Por tanto, los objetivos específicos de este trabajo han sido:

- Conocer y analizar los principios del olimpismo que han sido la base y han incidido a nivel práctico en la ayuda a las circunstancias de los refugiados.

- Conocer la presencia y la influencia de los principios olímpicos en la trayectoria olímpica de la deportista, Aauri Bokesa, hija de un exiliado político. 


\section{Metodología}

Teniendo en cuenta las circunstancias temporales en la que se centra el objeto de estudio de este trabajo, podemos afirmar que se trata de una investigación sobre la Historia del Tiempo Presente ya que con ella hemos reconstruido un aspecto parcial de la historia deportiva olímpica, relacionada con la aplicación de los principios olímpicos y su influencia en la realidad de los deportistas refugiados. Se trata de hechos históricos protagonizados por personas vivas que son fuentes primarias para una investigación histórica y que pueden ofrecernos su testimonio para reconstruir y rescatar un pequeño fragmento de la historia del deporte olímpico que sería imposible conocer a partir de otras fuentes.

Según afirma Martínez Gorroño (1996) la Historia del Tiempo Presente es la etapa de la Historia Contemporánea que cuenta con la circunstancia de que para su reconstrucción se puede contar con fuentes vivas. Ellas nos pueden ofrecen una información cualitativa de las circunstancias vividas que no es posible conocer a partir de fuentes escritas. Por otra parte, sobre la idoneidad del uso de la fuente oral para la investigación histórica, Adbón Mateos afirma que

«La fuente oral resulta una excelente vía de acceso a las nociones de memoria histórica colectiva y cultura política en la historia del tiempo presente. Su importancia se acrecienta en la medida en que el pasado reciente no haya cristalizado todavía como memoria autobiográfica. La debilidad de la literatura autobiográfica para el tiempo más inmediato solamente puede suplirse con la fuente oral. Acceder a la experiencia vivida autobiográfica y colectiva de un miembro de un grupo social o asociación humana en el tiempo presente depende, en buena medida, del recurso a la fuente oral». (Mateos López, A., 1998, p.3)

Como consecuencia de nuestra preparación metodológica, realizada paralelamente a los primeros pasos de esta investigación, hemos tomado conciencia según afirma Soto Gamboa de que el investigador de la Historia del Tiempo Presente «tiene la posibilidad de apreciar y observar las mentalidades, la percepción de los contemporáneos sobre el tema y sus propias vivencias». (Soto Gamboa, A., 2004, p.137)

El diseño metodológico que especialmente fuimos diseñando para el presente trabajo se inició con la utilización de la técnica conocida como Análisis Documental, que aplicamos a la fuente primaria que para este trabajo y su objeto de estudio supone la Carta Olímpica. De ella extrajimos la base de la filosofía olímpica; es decir, los principios y valores que han sido nuestro objeto de estudio, a fin de posteriormente comprobar su presencia en los contextos de la práctica olímpica en los que este trabajo se ha centrado.

Para obtener la primera información sobre el apoyo a los refugiados, es decir, el paso previo que supone «la aproximación al objeto de estudio», se utilizó información de una fuente secundaria: la página del Alto Comisionado de las Naciones Unidad para los Refugiados (ACNUR). Profundizando sobre la realidad de la práctica, hemos recabado información de los archivos de Comité Olímpico Internacional de Lausanna.

Las características de la metodología específica de la Historia del Tiempo Presente nos condicionaron a hacer previamente el trabajo de identificación y selección de una fuente primaria adecuada. Ella ha sido la deportista española Aauri Bokesa. Sus circunstancias de ser una mujer atleta de color, hija de un exiliado político y tener experiencia como participante en varias ediciones de los Juegos Olímpicos nos hicieron identificarla como una fuente primaria adecuada y pertinente, además de que se mostró fácilmente accesible. Posteriormente procedimos a la elaboración de un guión de una entrevista semiestructurada de final abierto, según las directrices y los aspectos metodológicos propuestos por Hammer, D. y Wildavsky, A. 
(1990). El contenido del guión de la entrevista se centraba en los resultados del análisis documental realizado previamente sobre la Carta Olímpica. El guión contenía preguntas concretas sobre los principios del ideario olímpico que de su contenido habíamos extraído y pretendíamos investigar sobre su presencia en su trayectoria deportiva olímpica.

Finalmente hemos recogido información de las fuentes hemerográficas que contenían noticias relativas al respecto de nuestro objeto de estudio.

Con los frutos obtenidos a partir de las técnicas y pesquisas ya expuestas, procedimos al contraste y triangulación de todas las informaciones, así como al proceso de crítica externa e interna de las fuentes y las informaciones, según las orientaciones y el proceso que propone Best, a fin de «establecer lo auténtico o genuino de los datos» (Best, 1982, p.81). Posteriormente elaboramos el relato histórico de los hechos que suponen los resultados de este trabajo de investigación y que son un pequeño fragmento de la historia del deporte olímpico actual y son un rescate de una parte de la Historia del Tiempo Presente, que evidencia la vigencia y presencia de los valores olímpicos en las circunstancias del Movimiento Olímpico actualidad.

\section{Los principios olímpicos en la práctica actual}

En los discursos y apariciones públicas del máximo representante del Movimiento Olímpico actual, Thomas Bach, siempre hace alusión e incide en la necesidad de la puesta en práctica de los principios del ideario olímpico e incita a su cumplimento y su extensión internacional en otros ámbitos. Así, por ejemplo, en su comparecencia ante la Asamblea General de las Naciones Unidas, celebrada en Nueva York el 26 de octubre de 2015, Bach decía textualmente en su "Discurso con motivo de la adopción de la resolución "construir un mundo mejor y más pacífico a través del deporte y del ideal olímpico»:

«En el deporte olímpico todo el mundo es igual, independientemente de su raza, sexo, condición social, contexto cultural, fe o religión. Este principio fundamental de no discriminación permite que el deporte promueva la paz y el entendimiento entre todos los pueblos.

Los Juegos Olímpicos son la culminación de esta visión. Durante los Juegos, los valores de tolerancia, solidaridad y paz cobran vida. La comunidad internacional se reúne para competir 'pacíficamente'...

En este espíritu olímpico de paz y solidaridad, el COI y la ACNUR asisten a refugiados en todo el mundo para llevarles actividad, esperanza y confianza en sí mismos a través del deporte». (Bach, T. 2016 pag.11).

\subsection{El equipo de refugiados}

Los principios fundamentales que suponen el ideario olímpico están recogidos en la Carta Olímpica. En ella misma se afirma que: «La Carta Olímpica, como instrumento de base de naturaleza constitucional, fija y recuerda los principios fundamentales y los valores esenciales del Olimpismo» (C.O. pag.9)

No obstante, cabe plantearse si estos principios son sólo una mera declaración de intenciones o si por el contrario son una realidad presente en la práctica y en las actividades que emprende el Movimiento Olímpico. En este sentido, hemos extraído de la Carta Olímpica a partir de la técnica conocida como «análisis documental» aquellos principios que nos parecen esenciales y que conforman su ideario y los valores que la filosofía olímpica pretende transmitir: la alegría del esfuerzo, el compromiso social, la solidaridad, y la no discriminación por razón de raza, religión, política y sexo. A partir de estos conceptos, fruto del análisis realizado, hemos 
estructurado la base de indagación sobre su presencia en la realidad práctica de ciertas acciones que en la actualidad se llevan a cabo, a partir de las estructuras del Movimiento Olímpico.

Así, a través de la información recabada en el Alto Comisionado de las Naciones Unidas para los Refugiados (ACNUR), junto a las aportaciones procedentes de los archivos del Comité Olímpico Internacional en Lausanne, podemos afirmar que dichos principios se han visto materializados en ciertas acciones que pasamos a enumerar. El COI, otorgó en 2016 becas a 10 deportistas de diferentes nacionalidades, sin distinción de raza, religión, política o sexo. Estas becas, financiadas por la Comisión del COI denominada Solidaridad Olímpica fueron adjudicadas con el objetivo de proporcionar a los deportistas refugiados una ayuda económica con el fin de que pudieran continuar sus entrenamientos y sus carreras deportivas, y convertirles en representantes y embajadores de los refugiados en varios eventos en todo el mundo. Los 10 deportistas que han sido los beneficiarios de estas becas son originarios de: Etiopía, Sudán del Sur, Siria y República Democrática del Congo. Los deportistas han sido víctimas de desplazamientos forzosos condicionados y obligados por la situación de inestabilidad política, guerras y por diferentes problemas sociales que han generado crisis de diferente índole en sus países de origen.

Durante los últimos Juegos Olímpicos de Río de Janeiro del 2016, el presidente del Comité Olímpico Internacional, Thomas Bach, se refirió en su discurso de apertura al equipo de refugiados como un símbolo de esperanza para todos los refugiados del mundo y como un medio para dar a conocer la magnitud de la crisis humanitaria que el desplazamiento forzado de personas está suponiendo en la actualidad. Desplazamientos forzados, que son la causa de múltiples sufrimientos, muertes y desgracias de inocentes de todo el mundo que se ven obligados a dejar sus lugares de residencia y sus formas de vida. Por otra parte, recordó que el espíritu olímpico se basa en la paz y en la solidaridad, por lo que el COI y ACNUR deben apoyar a los refugiados de todo el mundo para llevarles esperanza y confianza en sí mismos a través del deporte. Como prueba del resultado positivo de la creación de este equipo olímpico de refugiados podemos citar las declaraciones de Yusra Mardini, nadadora olímpica nacida en Siria, integrante del equipo de refugiados, quien afirmaba que no hablaban el mismo idioma, procedían de diferentes países, pero la bandera olímpica les unía, y, al fin y al cabo, representaban 60 millones de personas en todo el mundo.

Por otro lado, más allá de los Juegos Olímpicos, el COI sigue apoyando a los atletas a través del programa de apoyo a los refugiados puesto en marcha por Solidaridad Olímpica con el fin de ayudarles en su futuro, ya que las becas también pretenden contribuir a que los deportistas puedan formarse en una carrera profesional. Así, el programa les permite entrenarse, beneficiarse de una formación, ir a la universidad, integrarse en los países de acogida, además de, como ya hemos mencionado, participar en los últimos Juegos Olímpicos o en otras competiciones deportivas internacionales. También, el programa apoya y protege a un número determinado de atletas refugiados que aún viven en sus países de origen con el fin de prepararlos para las competiciones de alto nivel.

Finalmente, y siguiendo con el éxito de la participación del equipo olímpico de refugiados, el Comité Olímpico Internacional trabaja en el programa «Safe place» o «Lugares seguros». Dicho programa fue presentado por el Presidente del COI en la Asamblea General de la ONU en octubre de 2016. Esta iniciativa lanzada en cooperación con las Naciones Unidas permitirá mejorar la calidad de vida de los niños y adolescentes desplazados o en situación de riesgo creando lugares seguros para la práctica de deporte.

En definitiva, y remitiéndonos a la información recabada, se muestra como patente que los principios olímpicos, fundamentalmente los que hacen alusión a la solidaridad, el establecimiento de una sociedad pacífica, la responsabilidad social y la no discriminación, son 
una realidad que se ha materializado en el equipo olímpico de refugiados. Este hecho igualmente alcanza otro principio establecido y recogido en la Carta Olímpica «el valor educativo del buen ejemplo» (C.O. P. fundamental n. ${ }^{\circ}$ 1), ya que cabe reflexionar sobre el alcance y repercusión social que hoy suponen los Juegos Olímpicos. No olvidemos que los Juegos son uno de los más importantes eventos deportivos del mundo con mayor repercusión mediática y social a nivel mundial. En este marco de influencia social, el COI y ACNUR transmitieron en los Juegos de Río 2016 un mensaje al mundo de unidad, solidaridad y apoyo a todas aquellas personas que se ven obligadas a abandonar sus países de origen por conflictos armados o motivos políticos y sociales. Mensaje que se muestra como un «buen ejemplo» digno de ser imitado por otras instituciones. Por otra parte, es preciso incidir en la circunstancia de que este equipo está constituido por deportistas de muy diversas procedencias, de diferentes razas, religiones y sexo, y que supone además con todo ello, una muestra de la puesta en práctica de los principios fundamentales que hacen referencia a la no discriminación.

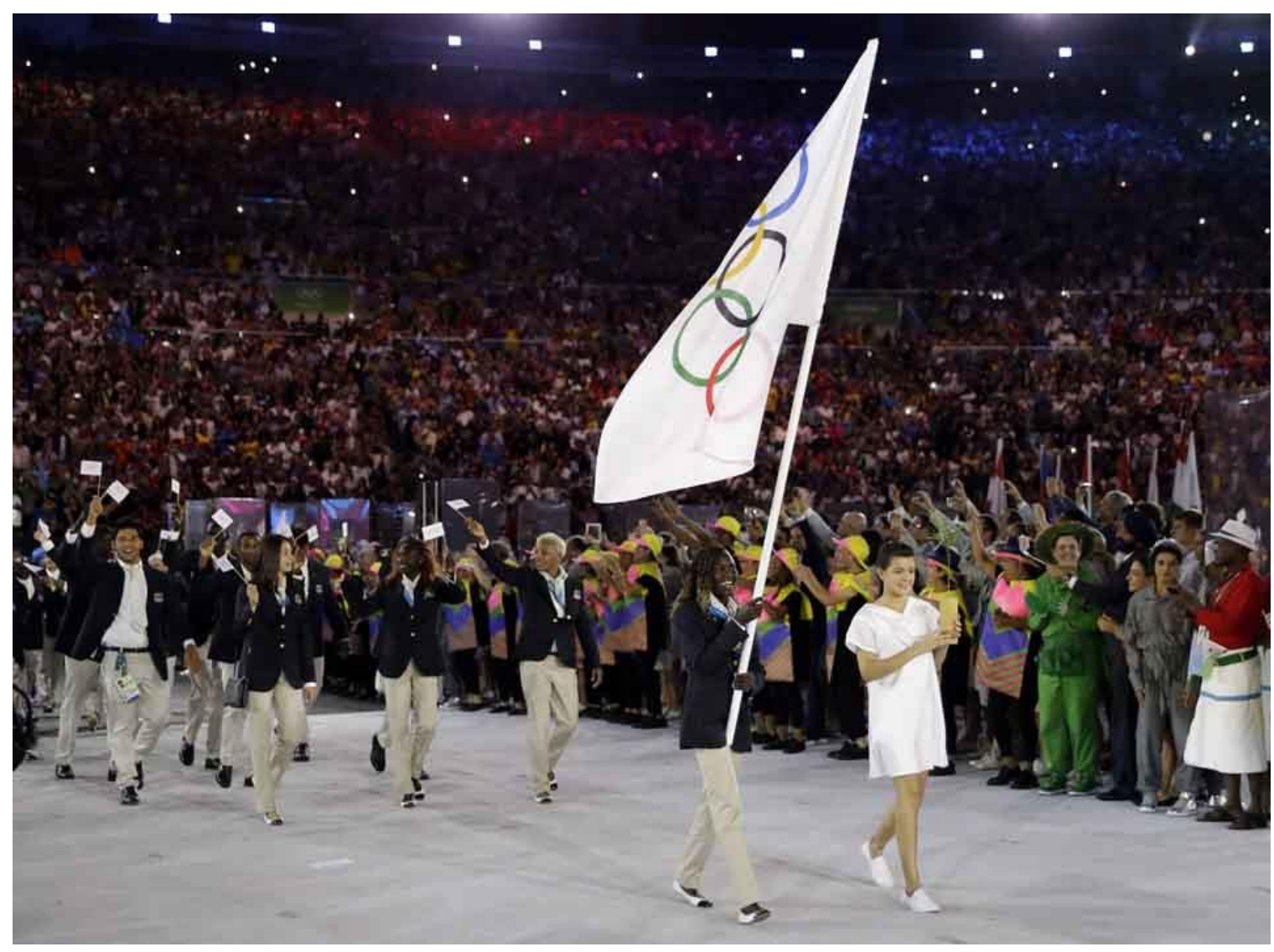

Foto 1. Equipo de refugiados durante la Ceremonia de Apertura de Los Juegos Olímpicos de Río 2016. Foto de archivo del Comité Olímpico Internacional.

\subsection{El ideario olímpico en la experiencia de Aauri Bokesa, deportista olímpica}

Aauri Bokesa, es una atleta española especialista en la prueba de 400 metros lisos. Ha sido seis veces campeona de España de 400m al aire libre, y cuatro veces campeona de España en la prueba de $400 \mathrm{~m}$, en pista cubierta. Ha participado en dos ediciones de los Juegos Olímpicos: Londres 2012 y Río 2016 formando parte del equipo olímpico español. En relación con su historia familiar cabe destacar el viaje de huida de su padre desde Guinea Ecuatorial hasta llegar a España pasando por varios países africanos y ubicándose por fin en España como refugiado político. Anacleto Bokesa es en la actualidad enfermero y Licenciado en Derecho por 
universidades españolas y tuvo que compaginar sus estudios con varios trabajos, lo que le requirió mucha dedicación y esfuerzo. Esta capacidad de esfuerzo y de sacrificio ha tenido una enorme influencia en la trayectoria deportiva de Aauri hasta el punto de llegar a convertirse en deportista olímpica y en uno de los grandes referentes del atletismo en España.

Como ya hemos indicado en el apartado del diseño metodológico, seleccionamos a Aauri Bokesa como fuente primaria para nuestro trabajo, por reunir varias circunstancias que la identificaban como especialmente idónea: es mujer, es negra, y es hija de Anacleto Bokesa, un exiliado político procedente de Guinea Ecuatorial que se vio obligado a abandonar Guinea, tras un golpe de estado y la imposición de una dictadura militar en su país, liderada por el teniente coronel Teodoro Obiang Nguema. La experiencia deportiva y de historia familiar de Aauri suponía un testimonio para la reconstrucción de un fragmento de la Historia del Tiempo Presente del deporte olímpico, que se nos mostraba como una oportunidad única para ser rescatada. Aunaba las vivencias deportivas dentro de las estructuras olímpicas, las circunstancias de su condición de mujer y de su raza y la influencia de una experiencia familiar caracterizada por la lucha, el esfuerzo y la defensa de los derechos humanos, en un colectivo familiar de desplazados por razones políticas.

En este sentido, y para recoger la opinión de Aauri Bokesa sobre la aplicación real de los principios olímpicos, como ya ha sido mencionado, llevamos a cabo una entrevista semiestructurada de final abierto. El diseño de su guión se estructuró en base a la síntesis de los principios y los valores identificados en el ideario olímpico, extraídos del análisis documental de la Carta Olímpica, del que partió la base de este trabajo de investigación. El guión contenía preguntas específicas para cada uno de los valores identificados a fin de recoger información sobre la presencia de cada uno de ellos en sus vivencias y experiencias como deportista presente en los Juegos Olímpicos; pero la semiestructuración de su guión permitía introducir preguntas espontáneas que podrían suscitarse tras ciertas informaciones que podría ofrecer la fuente oral, que hasta ese momento podrían ser conocidas o desconocidas para nosotros. Así, Hammer y Wildavsky afirman que «Habiéndose empapado del tema a estudiar, nuestro entrevistador intrépido es libre para probar las diversas y numerosas preguntas que le llevarán a asegurarse los resultados más relevadores». (Hammer y Wildavsky (1990, p.23)

Es importante reflexionar sobre que la circunstancia de la presencia de Aauri Bokesa en dos ediciones de los Juegos es un aspecto que le ha llevado a compartir no solo experiencias meramente deportivas con atletas, gestores y responsables de las estructuras olímpicas procedentes de todos los países, sino que le ha propiciado la oportunidad de compartir el ambiente cotidiano que se vive en la Villa Olímpica. Todos los allí presentes comparten residencia, comida, momentos de ocio, momentos de estrés, presiones de los medios de comunicación, etc. Nos interesaba especialmente rescatar de sus recuerdos si en su experiencia en aquel ambiente olímpico, y tras hacerle reflexionar específicamente sobre la filosofía olímpica, rememoraba un ambiente en el que la filosofía olímpica y sus valores estaban o no presentes.

A partir pues de su testimonio, podemos afirmar que Aauri Bokesa considera que la alegría del esfuerzo siempre la ha visto presente en el entorno del Movimiento Olímpico, y muy especialmente en el atletismo. Como consecuencia de tratarse de un deporte individual, es una especialidad que requiere aún más esfuerzo y más sacrificio si cabe que un deporte colectivo, pues en los deportes colectivos entiende que la capacidad de esfuerzo puede estar más repartida entre los jugadores. Las circunstancias específicas del atletismo también conllevan que el atleta consiga poder sentir una gran satisfacción por sus logros conseguidos con su empeño diario y sienta esa alegría del esfuerzo a la que hace alusión la Carta Olímpica. 
Durante su participación en los Juegos Olímpicos afirmaba que siempre ha visto cumplidos y presentes los principios olímpicos. Personalmente, no ha percibido ningún tipo de discriminación. Recordaba que, en alguna ocasión puntual, sí tenía constancias de que cuando ha habido algún comentario discriminatorio había sido duramente sancionado. En sus recuerdos al respecto, rememoró e hizo alusión a la exclusión de los Juegos Olímpicos de Londres 2012 de la saltadora griega Paraskevi quien realizó un comentario racista en una conocida red social.

A partir de su experiencia, consideraba que todos los valores se magnifican durante las competiciones y que otro principio, como es la solidaridad siempre se ha cumplido en los Juegos en los que ella ha participado. Nos expuso algunos ejemplos, como la foto realizada por dos deportistas de Korea del Norte y de Korea del Sur, o la imagen de las corredora Nikki Hamblin y Abbey D'Agostino ayudándose mutuamente para continuar la carrera tras las caídas durante una de las pruebas olímpicas. Afirma que las conductas antirreglamentarias son duramente sancionadas en el olimpismo, lo que permite que se desarrolle un juego más limpio y, en definitiva, más solidario.

Por otro lado, nunca se ha sentido discriminada dentro del Movimiento Olímpico por razones de raza. No obstante, en España, sí es cierto que en algunas ocasiones ha debido de justificar el hecho de formar parte del equipo olímpico español, por su color de piel, a pesar de haber nacido en este país. Tampoco se ha sentido discriminada por razón de sexo. Considera que el atletismo en España es un deporte muy igualitario puesto que los sueldos, las becas, los premios, y las pruebas son iguales, tanto para hombres como para mujeres. Por otra parte, y, además, los entrenamientos y los equipos son mixtos, aspecto que cree que potencia y estimula la igualdad. También afirmó que todas estas circunstancias están muy presentes en este deporte durante los Juegos Olímpicos.

Aauri nos dio también su opinión sobre el equipo de refugiados. Está muy de acuerdo y le parece muy positiva la creación de este equipo, ya que es una manera de dar voz a los refugiados y de visibilizar los problemas sociales a los que se debe dar respuesta. También piensa que este proyecto de ayuda a los refugiados debe extrapolarse a las demás instituciones de otros países y en todo tipo de competiciones.

Reconoce que existirán situaciones particulares u opiniones de deportistas que quizás no estén de acuerdo con ella, pero desde su experiencia sí que puede afirmar con rotundidad que, en su caso, y a partir de su experiencia en los Juegos Olímpicos, se han cumplido todos los principios del ideario olímpico.

En definitiva, Aauri Bokesa considera que los principios de la Carta Olímpica, aunque puedan parecer utópicos, son una realidad en el Movimiento Olímpico actual. Gracias al cumplimiento de dichos principios, el Movimiento Olímpico y concretamente los Juegos Olímpicos, adquieren las características de un proyecto internacional estrechamente relacionado con la cooperación para el desarrollo. 


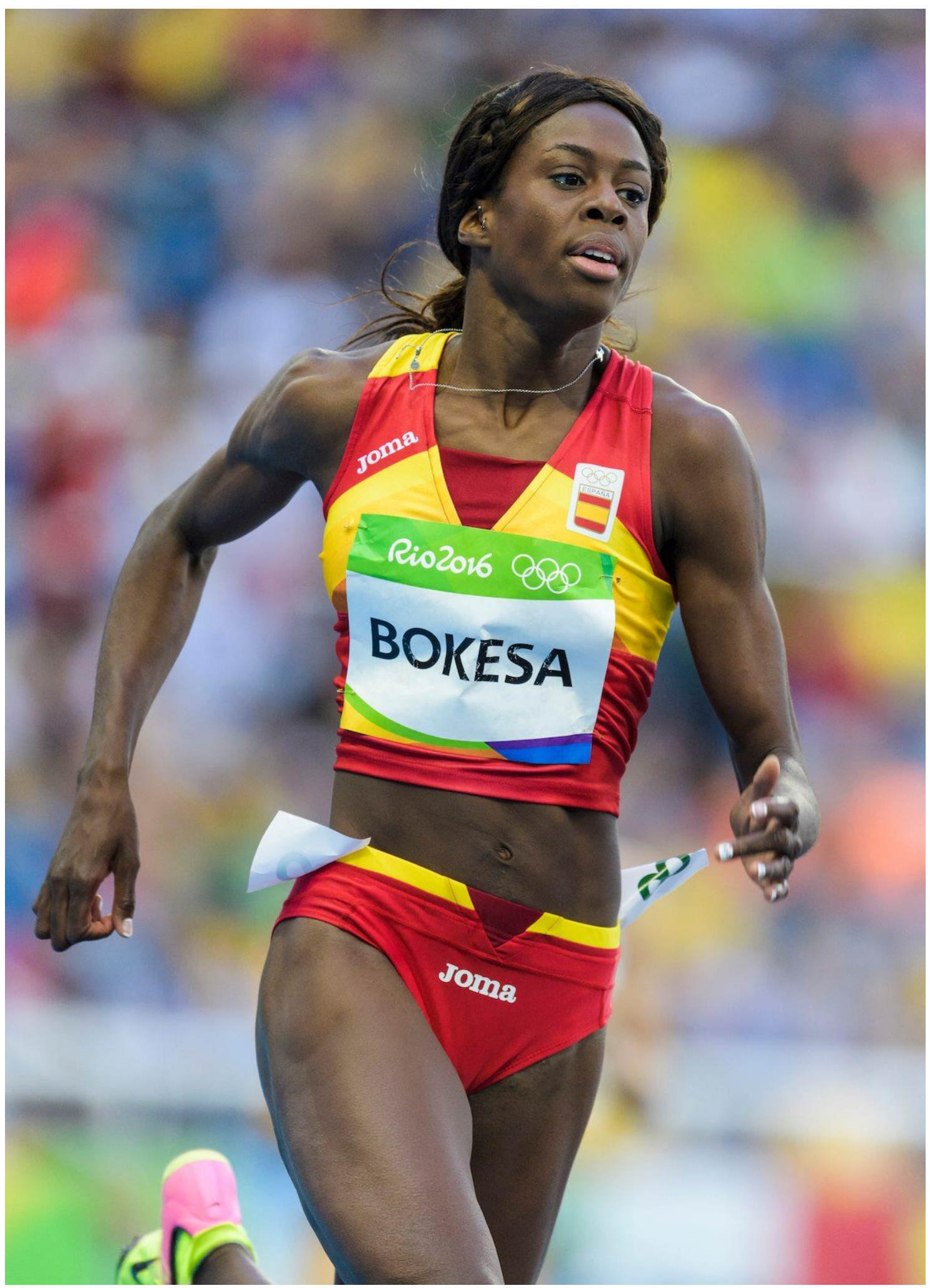

Foto 2. Aauri Bokesa durante su participación en Los Juegos Olímpicos de Río de Janeiro, 2016. Foto de archivo de Bokesa. 


\section{FUENTES Y BIBLIOGRAFÍA}

\subsection{Fuentes primarias}

\subsubsection{Fuente oral}

Aauri Bokesa. Atleta olímpica española. Entrevista realizada en Madrid el día 10 de Mayo de 2017

\subsubsection{Archivos y fuentes escritas}

- Archivo del Comité Olímpico Internacional. Lausana (Suiza)

- Olympic charter. (2015). Lausanne Switzerland. Consultado el 12/02/2016 en https://www.um.es/documents/933331/0/CartaOlimpica.pdf/8c3b36b2-11a2-4a77-876a41ae33c4a02b.

- IOC Media Relations Team: refugee olympic team a symbol of unifyng power of sport on international day of sport for developement and peace. 6/04/2017 https://www.olympic.org/news/refugee-olympic-team-a-symbol-of-unifying-power-ofsport-on-international-day-of-sport-for-development-and-peace

- Clayton, J and Godinho, L (22/08/2016) Historic Olympics come to an end in style for Team Refugees. Rio 2016: refuge olympic team. UNHCR.

- http://www.unhcr.org/news/latest/2016/8/57ba85674/historic-olympics-end-style-teamrefugees.html

- Comité Olympique International. L'équipe olympique de réfugiés, symbole d'espoir. (05/04/2017) https://www.olympic.org/fr/news/l-equipe-olympique-de-refugies-symbole-despoir

\subsection{Bibliografía}

- Bach, T. (2016). Discurso con motivo de la adopción de la resolución «Construir un mundo mejor y más pacífico a través del deporte y del ideal olímpico». Citius, Altius, Fortius. Humanismo, sociedad y deporte: investigaciones y ensayos, 9(1). Centro de Estudios Olímpicos de la Universidad Autónoma de Madrid. ISNN. 2340-9886. http://dx.doi.org/10.15366/citius2016.9.1.001

- Best, J.M., (1982). Cómo investigar en educación. Madrid: Ediciones Morata.

- Binder, D. (2007). Teaching values: an olympic education toolkit. A Project of the International Olympic Committe. Laussanne Switzerland.

- Calle-Molina, M y Martínez-Gorroño, M.E. (2014). Presencia o ausencia de los valores del movimiento olímpico en el currículo español de educación secundaria obligatoria en educación física: Un estudio de dos casos. Citius, Altius, Fortius. Humanismo, sociedad y deporte: investigaciones y ensayos.7, (1), 99-121. Centro de Estudios Olímpicos de la Universidad Autónoma de Madrid. 
- Fazio Vengoa, H. (1998). La historia del tiempo presente: una historia en construcción. Historia Crítica, 17, 47-57. Recuperado de http://www.redalyc.org/articulo.oa?id=81111329004

- Hammer, D. y Wildavsky, A. (1990). La entrevista semi-estructurada de final abierto. Aproximación a una guía operativa. Historia y Fuente Oral, 4, 23-61.

- Soto Gamboa, A. (2004). Historia del presente: estado de la cuestión y conceptualización. HAOL, 3, 101-116. Recuperado de:

http://dialnet.unirioja.es/descarga/articulo/829443.pdf

- Sauvage, P. (1988). Una historia del tiempo presente. Historia Crítica (17) 59-70. Recuperado de http://www.redalyc.org/articulo.oa?id=811113290045

- Martínez Gorroño, M. E. (1996). Metodología y fuentes en el estudio del exilio español en Colombia a consecuencia de la guerra civil 1936-1939. En Trujillano Sánchez J. M. y Díaz Sánchez P. Actas y Jornadas Historia y Fuentes orales-testimonios orales y escritos (pp. 253-258). Ávila, España: Fundación Cultural Santa Teresa.

- Mateos López, A. (1998). Historia, Memoria, Tiempo Presente. Hispania Nova, 1. Recuperado de http://hispanianova.rediris.es/general/articulo/004/art004.htm 